\title{
Magnitude of Multidrug Resistance among Bacterial Isolates from Surgical Site Infections in Two National Referral Hospitals in Asmara, Eritrea
}

\author{
Eyob Yohannes Garoy, ${ }^{1}$ Yacob Berhane Gebreab, ${ }^{2}$ Oliver Okoth Achila, ${ }^{1}$ \\ Nobiel Tecklebrhan, ${ }^{2}$ Hermon Michael Tsegai, ${ }^{2}$ Alex Zecarias Hailu, ${ }^{2}$ \\ Abrehet Marikos Buthuamlak, ${ }^{2}$ Tewelde Ghide Asfaga, \\ and Mohammed Elfatih Hamida $\left.{ }^{1}\right)^{1}$ \\ ${ }^{1}$ Department of Clinical Laboratory Sciences, Orotta College of Medicine and Health Sciences (OCMHS), Asmara, Eritrea \\ ${ }^{2}$ Department of Clinical Laboratory Sciences, Asmara College of Health Science (ACHS), Asmara, Eritrea
}

Correspondence should be addressed to Mohammed Elfatih Hamida; mohelfatih77@gmail.com

Received 10 November 2020; Revised 2 February 2021; Accepted 14 February 2021; Published 26 February 2021

Academic Editor: Joseph Falkinham

Copyright ( 2021 Eyob Yohannes Garoy et al. This is an open access article distributed under the Creative Commons Attribution License, which permits unrestricted use, distribution, and reproduction in any medium, provided the original work is properly cited.

\begin{abstract}
Background. The World Health Organization has emphasized the importance of understanding the epidemiology of MDR organisms from a local standpoint. Here, we report on a spectrum of bacteria associated with surgical site infections in two referral hospitals in Eritrea and the associated antibiotic susceptibility patterns. Methods. This survey was conducted between February and May 2017. A total of 83 patients receiving treatment for various surgical conditions were included. Swabs from infected surgical sites were collected using Levine technique and processed using standard microbiological procedures. In vitro antimicrobial susceptibility testing was performed on Mueller-Hinton Agar by the Kirby-Bauer disk diffusion method following Clinical and Laboratory Standards Institute guidelines. The data were analyzed using SPSS version 20. Results. A total of 116 isolates were recovered from 83 patients. In total, 67 (58\%) and 49 (42\%) of the isolates were Gram-positive and Gram-negative bacteria, respectively. The most common isolates included Citrobacter spp., Klebsiella spp., Escherichia coli, Proteus spp., Pseudomonas aeruginosa, Salmonella spp., Enterobacter spp., and Acinetobacter spp. In contrast, Staphylococcus aureus, CONS, and Streptococcus viridians were the predominant Gram-positive isolates. All the Staphylococcus aureus isolates were resistant to penicillin. MRSA phenotype was observed in $70 \%$ of the isolates. Vancomycin, clindamycin, and erythromycin resistance were observed in $60 \%, 25 \%$, and $25 \%$ of the isolates, respectively. Furthermore, a high proportion (91\%) of the Gram-negative bacteria were resistant to ampicillin and $100 \%$ of the Pseudomonas aeruginosa and Escherichia coli isolates were resistant to $>5$ of the tested antibiotics. The two Acinetobacter isolates were resistant to $>7$ antimicrobial agents. We also noted that 4 (60\%) of the Klebsiella isolates were resistant to $>5$ antimicrobial agents. Possible pan-drug-resistant (PDR) strains were also isolated. Conclusion. Due to the high frequency of MDR isolates reported in this study, the development and implementation of suitable infection control policies and guidelines is imperative.
\end{abstract}

\section{Background}

Surgical site infections (SSIs), a subcategory of nosocomial infections or healthcare-associated infections, are regarded as an important adverse postoperative event [1]. In terms of frequency and costs, a report from the USA indicated that
SSIs are second in frequency and third in cost [1]. The predominant bacterial isolates in hospital-acquired SSIs include Staphylococcus aureus, Enterococcus spp., Citrobacter spp., Pseudomonas aeruginosa, Klebsiella pneumoniae, Escherichia coli, and other Enterobacteriaceae. Acinetobacter baumannii is another bacterium that is 
causing increasing concern in different hospital departments (internal medicine, intensive care units (ICUs), surgery) and long-term-care settings [2,3].

Importantly, molecular profiles of isolates from apparently unrelated infections have reported widespread interspecies transfer of genes encoding antibiotic resistance, adding another facet to the threat posed by these organisms [1]. In fact, a high level of resistance to the commonly prescribed antibiotics like penicillin, ampicillin, tetracyclines, trimethoprim-sulfamethoxazole (TMP-SMX), chloramphenicol, and third-generation cephalosporins (cefoxitin, cefotaxime, and ceftriaxone) has been described in multiple countries in subSaharan Africa (SSA) [4-7].

In general, multidrug resistance (MDR) (concomitant resistance to $\geq 3$ different antimicrobial classes) is a common trait in these isolates $[1,6,8,9]$. In particular, extended spectrum- $\beta$-lactamases (ESBLs, class A) and AmpC $\beta$-lactamases (class C)-producing Escherichia coli and Klebsiella species have been described [6]. Previous reports of isolates that are resistant to second-generation cephalosporins and $\beta$-lactam/ $\beta$-lactamase inhibitor combinations in the absence of ESBL-containing plasmids exist [10]. In addition, healthcare-associated methicillin-resistant Staphylococcus aureus (HA-MRSA) oxacillin/nafcillin-resistant Staphylococcus aureus (ORSA); penicillin-resistant Streptococcus pneumoniae (PRSP); vancomycin-resistant enterococci (VRE), and carbapenem-resistant and fluoroquinolone-resistant Pseudomonas aeruginosa and Enterobacteriaceae have also emerged as important families of healthcare-associated pathogens worldwide $[6,11]$. Overall, multidrug-resistant (MDR) isolates complicate treatment and often result in extended hospital stay and higher morbidity and mortality [1].

At present, the emergence and rapid global spread of MDR threatens the gains made in the previous decades on the treatment and control of multiple bacterial infections. Importantly, multiple reports have consistently demonstrated that the frequency of MDR-associated SSI has increased globally over the last decade $[1,6,12]$ with low- and mediumincome countries (LMICs) reporting comparatively higher frequencies $[8,11,13]$. In Eritrea, a recent multicenter study noted that the frequency of HA-MRSA in hospitalized patients with wound infections was 72\% [14]. Altogether, existing data suggest a highly heterogeneous picture with significant intra- and intercountry variation potentially reflecting the multiplicity of infection control practices (or lack thereof) and antimicrobial prescription patterns within the region $[1,9]$.

Although the significance of gathering accurate data on resistance to antibacterial agents is well recognized, the problem is largely underinvestigated and/or underreported in LMICs in SSA. For instance, obtaining adequate countryspecific data on multiple facets of SSI is difficult despite the rapid expansion in facilities offering complex surgical procedures in urban and rural settings in SSA. And where data exists, it is often suboptimal/partial or conflicting. In Eritrea, data on the antimicrobial profiles of GNB or SSIs are lacking in international literature. In this regard, the true caseload of hospital-acquired SSIs in Eritrea is largely unknown. Adding to this concerning phenomenon is the fact that existing infection control practices in the country are largely rudimentary. To illustrate, the country has no infrastructure of trained infection-control professionals and lacks a laboratory-based surveillance and reporting system. Internment of multiple patients (often in close proximity) in single wards also limits the utility of contact precaution procedures. Moreover, antimicrobial therapy is infrequently guided by laboratory-generated drug susceptibility results or antibiotic stewardship programs.

Therefore, this study was designed to collect data on the spectrum of bacteria associated with SSIs in two referral hospitals in Eritrea. Phenotypic antibiotic resistance patterns were also profiled. Understanding the overall epidemiology of SSI from a local standpoint may be useful in guiding SSIs therapy and infection-control interventions such as screening and contact precautions, among others. The study may also provide crucial data on the magnitude of the problem in the country.

\section{Methods}

2.1. Study Design and Settings. This survey was conducted between February and May 2017 in two referral hospitals (Halibet National Referral Hospital and Orotta National Referral Hospital) in Asmara, Eritrea.

2.2. Study Population and Patient Recruitment. Male and female patients admitted in the hospital surgical wards after surgery were included in the study. Mostly, they were patients who developed infection after 48 hours of admission. Common indications included amputations, colonoscopies, appendectomies, and fixations associated with compound fractures and surgical debridement,among others. Pediatric patients, receiving treatment for various surgical conditions (and meeting the pre-set criteria), were also included. In this situation, consent to participate in the study was obtained from the parents.

2.3. Sample Collection and Processing. The infected sites (wound bed) were prepared prior to specimen collection by using Levine's technique [15]. The technique outlines the appropriate wound preparation procedures that should precede sample collection. To collect samples, a sterile cotton-tipped applicator was rotated gently over $1 \mathrm{~cm}^{2}$ area for 5 seconds. In the process, the pressure applied was sufficient to express the purulent exudates. Double wound swabs were subsequently taken from each wound.

\subsection{Laboratory Procedures}

2.4.1. Identification of Isolates. Specimens (wound swabs) were processed at the National Health Laboratory Bacteriology Laboratory within 1 hour after collection. One swab was used to make Gram stain smears. The other swab was inoculated into MacConkey agar (Oxoid, United Kingdom), chocolate agar, and mannitol salt agar. All cultures were incubated aerobically at $35-37^{\circ} \mathrm{C}$ for $24-48$ hours. Routine 
laboratory bench procedures including morphological characterization and biochemical tests (coagulase test, catalase reaction, mannitol fermentation, and deoxyribonuclease (DNase test)) were subsequently performed. Identification of GNB was carried out via the use of colony morphology on MacConkey and biochemical tests including triple sugar iron (TSI), urease test, citrate tests, and sulfur indole and motility (SIM) tests.

2.4.2. Drug Susceptibility Tests. Minimum inhibition concentrations (MICs) for a standard panel of antibiotics were evaluated on the basis of breakpoints set by the 2017 Clinical \& Laboratory Standards Institute standards (CLSI M100-S25) [16]. In this process, test and control organisms were first suspended in normal saline to McFarland 0.5 standard. The suspensions were seeded onto Mueller-Hinton agar (MHA) (Oxoid, UK). The disks to be tested were subsequently placed onto the media and incubated at $37^{\circ} \mathrm{C}$ for $24 \mathrm{hrs}$. Antimicrobials tested against specific Gram-negative rods (GNRs) and other isolates included cefalexin; ceftriaxone $30 \mu \mathrm{g}$; ceftazidime $30 \mu \mathrm{g}$; tetracycline $30 \mu \mathrm{g}$; gentamicin $10 \mu \mathrm{g}$ and amikacin-30 $\mu \mathrm{g}$; trimethoprim/sulfamethoxazole (TMPSMX) (cotrimoxazole); chloramphenicol $30 \mu \mathrm{g}$; ampicillin $10 \mu \mathrm{g}$; nitrofurantoin $300 \mu \mathrm{g}$; and ciprofloxacin $5 \mu \mathrm{g}$.

Gram-positive isolates (mostly Staphylococcus aureus isolates) were tested for the following antimicrobials: vancomycin $30 \mu \mathrm{g}$; chloramphenicol; penicillin 10 units; trimethoprim/sulfamethoxazole $1.25 / 23.75 \mu \mathrm{g}$; ciprofloxacin $5 \mu \mathrm{g}$; gentamicin $10 \mu \mathrm{g}$; tetracycline class (tetracycline-30 $\mu \mathrm{g}$ ); nitrofurantoin $300 \mu \mathrm{g}$; clindamycin $2 \mu \mathrm{g}$; erythromycin $15 \mu \mathrm{g}$; oxacillin $30 \mu \mathrm{g}$; and rifampicin $5 \mu \mathrm{g}$. The antimicrobial disks used for the stated tests were sourced from Oxoid Ltd., England. These drugs were selected based on the national list of medicines (Eritrean Formulary) to treat bacterial infections. Prescription frequencies and overall availability were also considered. Susceptibility testing was not performed for all Gram-positive isolates.

2.4.3. Characterization of MDR, MRSA, and VRSA. Multidrug-resistant (MDR) was defined as nonsusceptibility to at least one agent in $\geq 3$ antimicrobial drug classes [17]. Potential methicillin-resistant Staphylococcus aureus (MRSA) resistance was typed using disk diffusion (Oxoid ${ }^{\mathrm{TM}}$ disks). The double-disk diffusion test ( $\mathrm{D}$ test) was also performed to evaluate clindamycin resistance when discrepant macrolide test results were obtained (e.g., erythromycin resistant and clindamycin susceptible). In this test, a $2 \mu \mathrm{g}$ clindamycin disk is placed in close proximity (20 mm apart) to a $15 \mu \mathrm{g}$ erythromycin disk and agar plate that has been inoculated with a staphylococcal isolate and incubated overnight. D-testpositive isolates will exhibit a flattening of the clindamycin zone adjacent to the erythromycin disk, thereby displaying the characteristic D-like pattern.

2.5. Quality Control. Reference strains including S. aureus (ATCC-25923), E. coli (ATCC-25922), and P. aeruginosa (ATCC-27853) were used to quality control microbiological procedures such as staining, biochemical identification procedures, and drug susceptibility testing.

2.6. Data Analysis. The data were analyzed using Statistical Package for Social Sciences (SPSS Inc., version 20.0; IBM, Chicago, IL, USA). Tables and figures were used to report the data. The 2015 Standards Institute (CLSI M100-S25) breakpoints were used for all antimicrobial agents. Test outcomes were reported either as susceptible (S) or intermediate (I) or resistant (R).

2.7. Ethical Considerations. Ethical approval for the study was obtained from the Eritrean Ministry of Health $(\mathrm{MOH})$ research ethical committee. In addition, requisite permission was obtained from the hospital director and local administration. Participants were recruited voluntarily following provision of information on the study objective, study procedures, possible adverse effects, and the right to refuse or terminate their participation in the study at any time/ stage. Information on the maintenance of data confidentiality and integrity was also provided. To ensure data confidentiality and privacy, personal identifiers such as names were not collected.

\section{Results}

3.1. General Characteristics of the Study Participants and Associated Surgical Procedures. This study enrolled 83 patients-25(30.1\%) were females and 58 (69.9\%) were males. The average age $( \pm S D)$ of the patients was $42.65 \pm 13.14$ years (minimum 2 and maximum 82 years). Additional information on age grouping is shown in Table 1. In terms of institutional distribution, $48(50.4 \%)$ of the patients were from the Halibet hospital and 35 (49.6\%) were from the Orotta hospital. Amputations were the most common surgical procedure $35(42.2 \%)$ followed by 19(22.9\%) surgical debridement; 8(9.6\%) fixation (e.g., open reduction and internal fixation (ORIF) and/or removal of internal fixation devices); and 14 (16.9\%) colonoscopy and appendectomy. Additional surgical procedures contributed $8(9.6 \%)$ of the reported proportions.

3.2. Bacteria Isolated. In this study, the most common isolates included Citrobacter spp. 15 (25\%), Klebsiella pneumoniae 10 (15.6\%), Escherichia coli 10 (15.6\%), Pseudomonas aeruginosa (8 (12.7\%), Salmonella spp. 6(9.52\%), Enterobacter spp. 5 (7.94), and Acinetobacter spp. 2 (3.17). In contrast, the most common Gram-positive bacteria isolates included Staphylococcus aureus 20 (40.8\%), CONS (19 (38.78\%), Gram-positive bacilli 7 (14.29\%), and Streptococcus viridans 3 (6.12\%) (Table 2). Overall, single bacterial isolates were recovered from $53(63.9 \%)$ patients, while the rest $30(36.1 \%)$ had polymicrobial infections.

3.3. Antimicrobial Resistance Pattern: Gram-Positive Isolates. The drug resistance profile of the 20 Staphylococcus aureus isolates was evaluated. In this analysis, $20(100 \%)$ of the 
TABLE 1: Characteristics of patients with positive culture from surgical wards in the Halibet and the Orotta National Referral Hospital in Asmara, Eritrea, 2017.

\begin{tabular}{|c|c|c|c|c|c|c|}
\hline \multirow{2}{*}{ Variable } & \multicolumn{5}{|c|}{ Type of surgery } & \multirow{2}{*}{ Total N (\%) } \\
\hline & Amputation $N(\%)$ & Col + Append $N(\%)$ & Debridement $N(\%)$ & Fixation $N(\%)$ & Others $N(\%)$ & \\
\hline \multicolumn{7}{|l|}{ Sex } \\
\hline Female & $8(32.0)$ & $6(24.0)$ & $5(20.0)$ & $1(4.0)$ & $5(20.0)$ & $25(30.1)$ \\
\hline Male & $26(44.8)$ & $8(13.8)$ & $14(24.1)$ & $7(12.1)$ & $3(5.2)$ & $58(69.9)$ \\
\hline \multicolumn{7}{|l|}{ Occupation } \\
\hline Farmer & $9(47.4)$ & $4(21.1)$ & $4(21.1)$ & $1(5.3)$ & $1(5.3)$ & $19(22.9)$ \\
\hline House wife & $7(43.8)$ & $3(18.8)$ & $2(12.5)$ & $1(6.2)$ & $3(18.8)$ & $16(19.3)$ \\
\hline Self-employed & $5(55.6)$ & $0(0.0)$ & $2(22.2)$ & $1(11.1)$ & $1(11.1)$ & $9(10.8)$ \\
\hline Student & $1(6.2)$ & $3(18.8)$ & $8(50.0)$ & $2(12.5)$ & $2(12.5)$ & $16(19.3)$ \\
\hline Others & $12(52.2)$ & $4(17.4)$ & $3(13.0)$ & $3(13.0)$ & $1(6.2)$ & $23(27.7)$ \\
\hline \multicolumn{7}{|l|}{ Hospital } \\
\hline Halibet & $19(39.6)$ & $2(4.2)$ & $15(31.2)$ & $8(16.7)$ & $4(8.3)$ & $48(58.8)$ \\
\hline Orotta & $15(42.9)$ & $12(34.3)$ & $4(11.4)$ & $0(0.0)$ & $4(11.4)$ & $35(42.2)$ \\
\hline \multicolumn{7}{|c|}{ Duration of operation } \\
\hline$<60$ minutes & $17(40.5)$ & $7(16.7)$ & $8(19.0)$ & $4(9.5)$ & $6(14.3)$ & $42(50.4)$ \\
\hline$>60$ minutes & $17(41.5)$ & 7 (17.1) & $11(26.8)$ & $4(9.8)$ & $2(4.9)$ & $41(49.6)$ \\
\hline \multicolumn{7}{|l|}{ Age of patient } \\
\hline$<18$ years & $2(12.5)$ & $4(25.0)$ & $4(25.0)$ & $3(18.8)$ & $3(18.8)$ & $16(19.3)$ \\
\hline $18-35$ years & $5(27.8)$ & $3(16.7)$ & $6(33.3)$ & $1(5.6)$ & $3(16.7)$ & $18(20.7)$ \\
\hline $36-60$ years & $13(50.0)$ & $4(15.4)$ & $5(19.2)$ & $2(7.7)$ & $2(7.7)$ & $26(31.3)$ \\
\hline$>60$ years & $14(60.9)$ & $3(13.0)$ & $4(17.4)$ & $2(8.7)$ & $0(0.0)$ & $23(27.7)$ \\
\hline
\end{tabular}

Col + Append: colostomy and appendectomy.

TABLE 2: Distribution of bacterial isolates in relation to type of operation among patients in surgical wards at the Halibet and the Orotta National Referral Hospital in Asmara, Eritrea, 2017.

\begin{tabular}{|c|c|c|c|c|c|c|}
\hline \multirow{2}{*}{ Variable } & \multicolumn{5}{|c|}{ Type of surgery } & \multirow{2}{*}{ Total $N(\%)$} \\
\hline & Amputation & Col + Append & Debridement & Fixation & Others & \\
\hline \multicolumn{7}{|l|}{ Gram-positive bacteria } \\
\hline Gram-positive bacilli & $4(57.14)$ & $1(14.29)$ & $1(14.29)$ & $1(14.29)$ & $0(0.0)$ & $7(14.29)$ \\
\hline CONS & $7(36.84)$ & $3(15.79)$ & $4(21.05)$ & $4(21.05)$ & $1(5.26)$ & $19(38.78)$ \\
\hline Staphylococcus aureus & $8(40.0)$ & $3(15.0)$ & $3(15.0)$ & $3(15.0)$ & $3(15.0)$ & $20(40.8)$ \\
\hline Streptococcus viridians & $1(33.33)$ & $1(33.33)$ & $1(33.33)$ & $0(0.0)$ & $0(0.0)$ & $3(6.12)$ \\
\hline Total N (\%) & $20(40.82)$ & $8(16.33)$ & $9(18.37)$ & $8(16.33)$ & $4(8.16)$ & $49(100)$ \\
\hline \multicolumn{7}{|l|}{ Gram-negative bacteria } \\
\hline Citrobacter spp. & $7(47.0)$ & $3(20.0)$ & $2(13.3)$ & $1(6.7)$ & $2(13.3)$ & $15(25.40)$ \\
\hline Proteus spp. & $3(37.5)$ & $0(0.0)$ & $3(37.5)$ & $0(0.0)$ & $2(25)$ & $8(12.7)$ \\
\hline Escherichia coli & $3(30.0)$ & $3(30)$ & $3(30)$ & $0(0.0)$ & $1(10)$ & $10(15.6)$ \\
\hline Klebsiella spp. & $2(20.0)$ & $1(10.0)$ & $5(50)$ & $2(20.0)$ & $0(0.0)$ & $10(15.6)$ \\
\hline Acinetobacter spp. & $1(50.0)$ & $0(0.0)$ & $1(50.0)$ & $0(0.0)$ & $0(0.0)$ & $2(3.17)$ \\
\hline Enterobacter spp. & $3(60.0)$ & $0(0.0)$ & $2(40.0)$ & $0(0.0)$ & $0(0.0)$ & $5(7.94)$ \\
\hline P. aeruginosa & $5(62.5)$ & $2(25.0)$ & $1(12.5)$ & $0(0.0)$ & $0(0.0)$ & $8(12.7)$ \\
\hline Salmonella spp. & $1(16.7)$ & $2(33.33)$ & $2(33.33)$ & $1(16.7)$ & $0(0.0)$ & $6(9.52)$ \\
\hline Total N (\%) & $25(39.1)$ & $11(17.2)$ & $19(30.0)$ & $4(6.25)$ & $5(7.8)$ & $64(100)$ \\
\hline
\end{tabular}

CONS: coagulase-negative staphylococci; $\mathrm{Col}+$ Append: colostomy and appendectomy.

isolates were resistant to penicillin, $14(70 \%)$ were resistant oxacillin, and $12(60 \%)$ were resistant to vancomycin. Similarly, 5 (25\%) were resistant to clindamycin. A similar proportion of isolates were resistant to Erythromycin (Figure 1). Resistance profiles for CONS, Streptococcus viridans, and Gram-positive bacilli were not evaluated.
3.4. Antimicrobial Susceptibility: Gram-Negative Isolates. The antimicrobial agents and number of proportions of isolates determined to be resistant and intermediate are presented in Table 3. The agent with the highest level of resistance was ampicillin with Citrobacter spp., Escherichia coli, Klebsiella spp., Enterobacter spp., and Pseudomonas spp. 


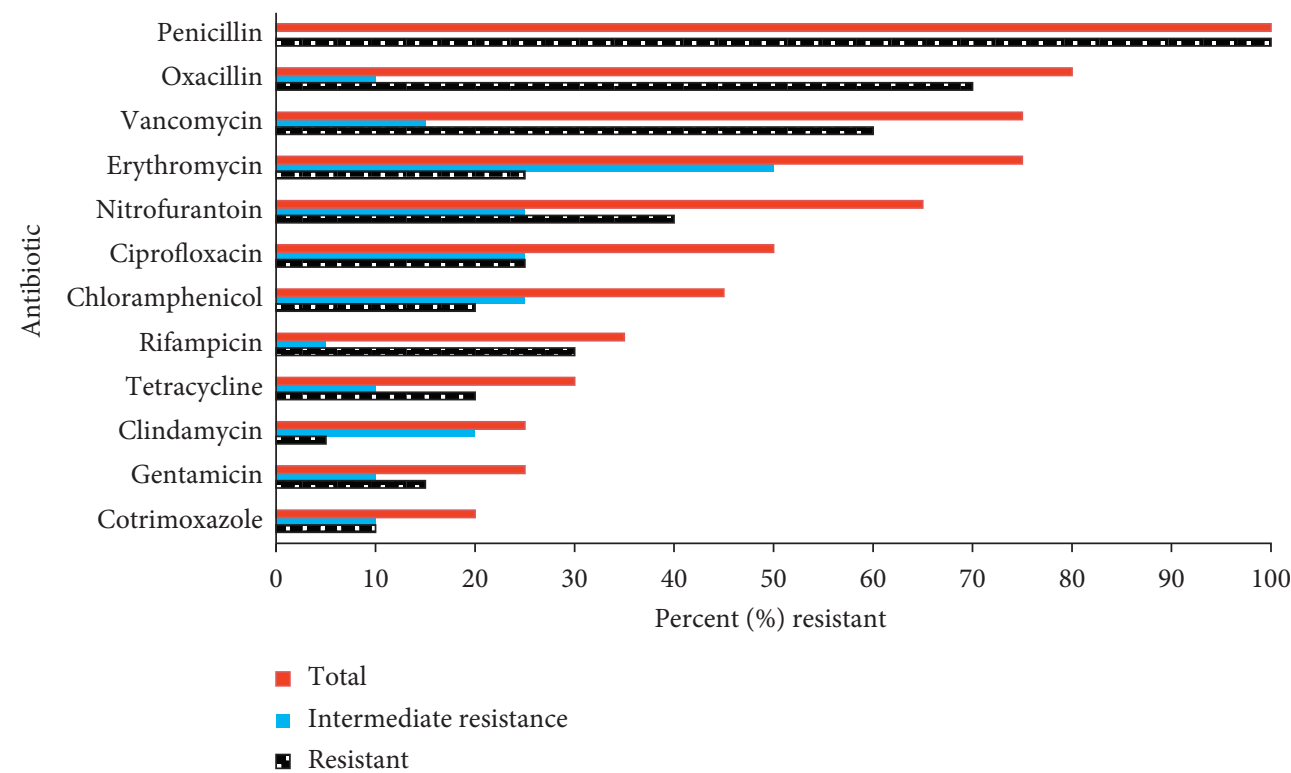

Figure 1: Disk diffusion test for Gram-positive isolates on 12 antibiotics, on Mueller-Hinton agar with $4 \% \mathrm{NaCl}$, as recommended in the Clinical and Laboratory Standards Institute guidelines.

Table 3: Disk diffusion test for Gram-negative isolates on 11 antibiotics, on Mueller-Hinton agar with $4 \% \mathrm{NaCl}$, as recommended in the Clinical and Laboratory Standards Institute guidelines.

\begin{tabular}{|c|c|c|c|c|c|c|c|c|c|c|c|c|c|}
\hline \multirow[b]{2}{*}{$\begin{array}{l}\text { Bacteria } \\
\text { isolated }\end{array}$} & & \multirow[b]{2}{*}{$\begin{array}{c}\text { Total } \\
\text { no. }\end{array}$} & \multicolumn{11}{|c|}{ Antimicrobial agent tested } \\
\hline & & & $\begin{array}{l}\text { C N } \\
(\%)\end{array}$ & $\begin{array}{c}\text { CIP } N \\
(\%)\end{array}$ & $\begin{array}{c}\text { SXT N } \\
(\%)\end{array}$ & $\begin{array}{c}\text { GN } N \\
(\%)\end{array}$ & $\begin{array}{l}\text { F N } \\
(\%)\end{array}$ & $\begin{array}{c}\text { TC } N \\
(\%)\end{array}$ & $\begin{array}{c}\text { Amp. } N \\
(\%)\end{array}$ & $\begin{array}{c}\text { CEP } N \\
(\%)\end{array}$ & $\begin{array}{c}\text { CFT } N \\
(\%)\end{array}$ & $\begin{array}{c}\text { CFR } N \\
(\%)\end{array}$ & $\begin{array}{c}\operatorname{Ami} N \\
(\%)\end{array}$ \\
\hline \multirow[t]{2}{*}{ Citrobacter spp. } & $\mathrm{R}$ & 15 & $6(40)$ & $7(47)$ & $9(60)$ & $10(67)$ & $\begin{array}{c}12 \\
(80)\end{array}$ & $11(73)$ & $15(100)$ & $14(93)$ & $14(93)$ & $11(73)$ & $3(20)$ \\
\hline & $\mathrm{I}$ & & $2(13)$ & 1( & $0(0)$ & 1 & $0(0)$ & 1 & & $0(0)$ & 1 & 1 & 7 \\
\hline \multirow{2}{*}{ Proteus spp. } & $R$ & 8 & $3(38)$ & $2(25)$ & $2(33)$ & $2(25)$ & $7(88)$ & $6(75)$ & $4(50)$ & $5(63)$ & $4(50)$ & $1(13)$ & $0(0)$ \\
\hline & $\mathrm{I}$ & & 0 & 0 & $0(0)$ & $0(0)$ & $0(0)$ & $0(0)$ & & $0(0)$ & $0(0)$ & $0(0)$ & 3 \\
\hline \multirow{2}{*}{ Escherichia coli } & $\mathrm{R}$ & 10 & $6(60)$ & $6(60)$ & $9(90)$ & $7(70)$ & $7(70)$ & $9(90)$ & $10(100)$ & $8(80)$ & $9(90)$ & $8(80)$ & $0(0)$ \\
\hline & $I$ & & $0(0)$ & 3 & $0(0)$ & $0(0)$ & $0(0)$ & $0(0)$ & & $0(0)$ & & $0(0)$ & 3 \\
\hline \multirow{2}{*}{ Klebsiella spp. } & $\mathrm{R}$ & 10 & $3(30)$ & $2(20)$ & $5(50)$ & $4(40)$ & $9(90)$ & $4(40)$ & $10(100)$ & $6(60)$ & $8(80)$ & $4(40)$ & $2(20)$ \\
\hline & $I$ & & $0(0)$ & $0(0)$ & 1 & 1 & $0(0)$ & 2 & $0(0)$ & $0(0)$ & $0(0)$ & $0(0)$ & $3(38)$ \\
\hline \multirow{2}{*}{$\begin{array}{l}\text { Acinetobacter } \\
\text { spp. }\end{array}$} & $R$ & 2 & $1(50)$ & $1(50)$ & $1(50)$ & $1(50)$ & $2(100)$ & $2(100)$ & $2(100)$ & $2(100)$ & $2(100)$ & $2(100)$ & $0(0)$ \\
\hline & $I$ & & 1 & $0(0)$ & $0(0)$ & 1 & $0(0)$ & $0(0)$ & $0(0)$ & $0(0)$ & $0(0)$ & $0(0)$ & $0(0)$ \\
\hline \multirow{2}{*}{$\begin{array}{l}\text { Enterobacter } \\
\text { spp. }\end{array}$} & $\mathrm{R}$ & 5 & $1(20)$ & $0(0)$ & $2(40)$ & $1(20)$ & $4(80)$ & $0(0)$ & $5(100)$ & $3(60)$ & $4(80)$ & $0(0)$ & $1(20)$ \\
\hline & $I$ & & $0(0)$ & 2 & $0(0)$ & 1 & $0(0)$ & 2 & & $0(0)$ & 1 & 1 & $0(0)$ \\
\hline \multirow{2}{*}{$\begin{array}{l}\text { Pseudomonas } \\
\text { spp. }\end{array}$} & $\mathrm{R}$ & 8 & $4(50)$ & $2(25)$ & $5(63)$ & $1(13)$ & $8(100)$ & $7(88)$ & $8(100)$ & $8(100)$ & $8(100)$ & $8(100)$ & $2(20)$ \\
\hline & $I$ & & 1 & $0(0)$ & 2 & 1 & $0(0)$ & 1 & $0(0)$ & $0(0)$ & $0(0)$ & $0(0)$ & 1 \\
\hline \multirow{2}{*}{ Salmonella spp. } & $R$ & 6 & $4(67)$ & $0(0)$ & $2(33)$ & $2(33)$ & $5(83)$ & $2(33)$ & $4(67)$ & $5(83)$ & $3(50)$ & $2(33)$ & $0(0)$ \\
\hline & $I$ & & $0(0)$ & $0(0)$ & $0(0)$ & $0(0)$ & 1 & 2 & & 2 & $0(0)$ & $0(0)$ & $0(0)$ \\
\hline Total & & 64 & $\begin{array}{c}28 \\
(44)\end{array}$ & $20(31)$ & $35(54)$ & $28(43)$ & $\begin{array}{c}54 \\
(85)\end{array}$ & $41(64)$ & $58(91)$ & $51(80)$ & $52(80)$ & $36(55)$ & $8(12)$ \\
\hline
\end{tabular}

C: chloramphenicol; CIP: ciprofloxacin; SXT: trimethoprim-sulfamethoxazole; GN: gentamicin; F: nitrofurantoin; TC: tetracycline; Amp: ampicillin; Cep: cefalexin; CFT: ceftazidime; CFR: ceftriaxone; Ami: amikacin.

isolates exhibiting $100 \%$ resistance. The highest susceptibility rates recorded for Gram-negative bacteria (with the exception of Klebsiella) were to amikacin.

Escherichia coli isolates exhibited high resistance $(>60 \%$ resistance) to chloramphenicol, ciprofloxacin, trimethoprimsulfamethoxazole, gentamicin, nitrofurantoin, tetracycline, cefalexin, ceftazidime, and ceftriaxone. However, no resistance was observed for amikacin. Similarly, Klebsiella spp. isolates exhibited high resistance $(>60 \%)$ to nitrofurantoin, cefalexin, ceftazidime, and ceftriaxone. High level of resistance (80\% resistance) was also observed for Pseudomonas spp. isolates to nitrofurantoin, tetracyclines, cefalexin, ceftazidime, and ceftriaxone. Citrobacter spp. isolates had $>70 \%$ resistance to several agents including tetracyclines, cefalexin, ceftazidime, and ceftriaxone. The two Acinetobacter isolates had $100 \%$ resistance to nitrofurantoin, tetracycline, cefalexin, 
ceftazidime, and ceftriaxone. See Figure 1 for additional resistance profiles.

3.5. Multidrug-Resistant (MDR) Patterns of Gram-Negative Isolates. The MDR patterns of specific isolates are presented in Table 4. In this analysis, 65 (97\%) of the isolates were resistant to $\geq 3$ classes of antimicrobial agents with varying combination of agents. In general, $8(100 \%)$ of the Pseudomonas aeruginosa were resistant to $>6$ of the tested agents. Similarly, $10(100 \%)$ of the Escherichia coli isolates were resistant to $>5$ agents. The two Acinetobacter isolates were resistant to $>7$ antimicrobial agents. We also noted that 4 (60\%) of the Klebsiella isolates were resistant to $>5$ antimicrobial agents. The proportion of Salmonella isolates which were resistant to $>6$ antimicrobial agents was $67 \%$.

3.6. Multidrug-Resistant (MDR) Patterns of Staphylococcus aureus. Analysis of the combinations of antimicrobial agent-specific isolates of Staphylococcus aureus that were susceptible are presented in Table 5. Results indicate that all the isolates were resistant to penicillin. At the same time, 17 $(85 \%)$ of the isolates were resistant to $>2$ classes of antimicrobial agents. The proportion of isolates which were identified as MRSA was 14 (70). For MRSA, high sensitivity to clindamycin, erythromycin, trimethoprim-sulfamethoxazole, and gentamicin was observed. The proportion of MRSA which were resistant to vancomycin was 9 (64\%).

\section{Discussion}

In hospital wards in SSA, the risk of nosocomial infections (SSIs, in particular) is often high $[7,9,11,12,18,19]$. Multiple reports from the region have implicated a variety of GNB as the dominant agents. In this study, the most common isolates included Citrobacter spp. (25.4\%), Klebsiella spp. (15.87\%), Escherichia coli (12.7\%), Proteus spp. (12.7\%), Salmonella spp. (12.7\%), Enterobacter spp. (7.94\%), and Acinetobacter spp. (3.17\%). A significant number of Pseudomonas aeruginosa isolates were also present (12.7\%). In contrast, Gram-positive bacteria isolates were predominantly Staphylococcus aureus (40.8\%), CONS (38.78\%), Gram-positive bacilli (14.29\%), and Streptococcus viridians (6.12\%). The dominance of these isolates in SSIs has been reported by multiple investigators in the region. The findings from this study support evidence that despite significant intra- and/or intercountry variation along with institutional variation, Klebsiella spp., E. coli, Proteus spp., and Pseudomonas aeruginosa typically switch places between the first, second, third, and fourth most commonly isolated GNB in a majority of settings in SSA $[4,9,18,20]$.

The predominance of the relatively uncommon Citrobacter spp. in this setting is notable. However, high frequencies of Citrobacter spp. isolates have been reported in some studies from the region. For instance, a study conducted in an intensive care unit in Kenyatta National Hospital (one of the largest referral hospitals in East and Central Africa) reported high frequency of $P$. aeruginosa, Klebsiella, Citrobacter spp., and S. aureus [9]. The low frequency of Acinetobacter spp. isolates is also in harmony with some reports from the region [9]. In contrast, the dominant Gram-positive isolate was Staphylococcus aureus. Beyond the current results, the normal flora nature of some of these isolates on skin and mucosal surfaces along with the acquisition of diverse extraintestinal virulence factors and AMR mechanisms provides possible justification for their emergence as a threat to postoperative and ICU patients.

In vitro analysis of AMR in multiple GNBs noted a high level of resistance to commonly used antibiotics like ampicillin, trimethoprim-sulfamethoxazole, and tetracycline. Resistance of specific isolates to cefalexin, ceftriaxone, and ceftazidime was also high. To illustrate, the $E$ coli isolated in this study were $100 \%$ resistant to ampicillin and $>80 \%$ trimethoprim-sulfamethoxazole, tetracycline, cefalexin, ceftriaxone, and ceftazidime. High resistance $(>80 \%)$ to multiple antibiotics (ampicillin, nitrofurantoin, and ceftriaxone) was also observed for Klebsiella pneumoniae isolates. This result is in harmony with some reports from the region [21]. Sensitivity of the Klebsiella pneumoniae isolates to amikacin and ciprofloxacin was also high. Furthermore, the isolated Pseudomonas aeruginosa were 100\% resistant to nitrofurantoin, ampicillin, ceftriaxone, and ceftazidime. High level of resistance to tetracycline and trimethoprimsulfamethoxazole was also noted in this isolates. Another atypical outcome observed in this study was the high resistance of most isolates to nitrofurantoin-an antibiotic indicated for urinary tract infections (UTIs) and for prophylactic use in recurrent UTI. An explanation for this outcome is unclear.

A number of issues are notable from these results. On the whole, the extensive postprocedural contamination of surgical sites by enterobacterial isolates is concerning. In particular, the high proportion of third-generation cephalosporin-resistant Escherichia coli (G3CREC) should raise concern, given its potential for pathogenicity [22]. This observation is consistent with previous studies in SSA, which have repeatedly noted that the frequency of G3CREC in the region is disproportionately high [23-25]. Emphasizing these concerns, a recent report noted that resistance to cephalosporins are increasing among nosocomial and community-acquired strains of GNBs worldwide [4] and that the high $\mathrm{BoD}$ associated with these strains (including G3CREC) will inevitably increase [22].

Multiple drivers of the projected increase exist. According to some reports, imprudent antibiotic use and poor adherence to infection-control practices are the keystone issues [26]. In SSA, these fundamental issues are poorly documented. In Eritrea for instance, there are no reports on the level of compliance by hospitals/healthcare workers to transmission-based control guidelines such as active surveillance to identify colonized patients and workers, decolonization of patients and healthcare workers, environmental decontamination or the widespread use of rub-in hand disinfection techniques. Research on the effectiveness of infection control practices (decontamination, disinfection, and sterilization) is particularly lacking. Another driver of the observed outcome is the extensive institutional use of cephalosporins in preoperative antibiotic 
TABLE 4: Multidrug resistance patterns of Gram-negative isolates among patients in surgical wards at the Halibet and the Orotta National Referral Hospital in Asmara, Eritrea, 2017.

\begin{tabular}{|c|c|c|c|c|c|c|c|c|c|c|}
\hline $\begin{array}{l}\text { Resistant } \\
\text { patterns of } \\
\text { antibacterial } \\
\text { agents tested }\end{array}$ & $\begin{array}{l}\text { No. of } \\
\text { agents }\end{array}$ & $\begin{array}{l}\text { Klebsiella } \\
\text { spp., } N \\
(\%)\end{array}$ & $\begin{array}{l}\text { Pseudomonas } \\
\text { spp., } N(\%)\end{array}$ & $\begin{array}{l}\text { Acinetobacter } \\
\text { spp., } N(\%)\end{array}$ & $\begin{array}{l}\text { Enterobacter } \\
\text { spp., } N(\%)\end{array}$ & $\begin{array}{l}\text { Citrobacter } \\
\text { spp., } N(\%)\end{array}$ & $\begin{array}{l}\text { Proteus } \\
\text { spp., } N \\
(\%)\end{array}$ & $\begin{array}{l}\text { Salmonella } \\
\text { spp., } N(\%)\end{array}$ & $\begin{array}{l}\text { Escherichia } \\
\text { coli, N (\%) }\end{array}$ & $\begin{array}{c}\text { Total } \\
\text { no. }(\%)\end{array}$ \\
\hline $\begin{array}{l}\text { C, CIP, SXT, GN, } \\
\text { F, TC, AMP, CEP, } \\
\text { CFR, CFT }\end{array}$ & 10 & - & - & - & - & $4(26.7)$ & - & - & $3(30)$ & $\begin{array}{c}6 \\
(10.45)\end{array}$ \\
\hline $\begin{array}{l}\text { C, CIP, SXT, F, } \\
\text { TC, AMP, CEP, } \\
\text { CFR, CFT, AMI } \\
\end{array}$ & 10 & - & $1(12.5)$ & - & - & - & - & - & - & $1(1.49)$ \\
\hline $\begin{array}{l}\text { C, CIP, GN, F, } \\
\text { TC, AMP, CEP, } \\
\text { CFR, CFT } \\
\end{array}$ & 9 & - & - & $1(50)$ & - & - & - & - & $1(10)$ & $2(2.99)$ \\
\hline $\begin{array}{l}\text { C, CIP, SXT, GN, } \\
\text { F, TC, Amp, CEP, } \\
\text { CFT }\end{array}$ & 9 & $2(20)$ & - & - & - & - & - & - & - & $2(2.99)$ \\
\hline $\begin{array}{l}\text { C, CIP, SXT, GN, } \\
\text { F, TC, AMP, CFR, } \\
\text { CFT }\end{array}$ & 9 & - & - & - & - & - & $1(12.5)$ & - & - & $1(1.49)$ \\
\hline $\begin{array}{l}\text { C, CIP, SXT, GN, } \\
\text { TC, AMP, CEP, } \\
\text { CFR, CFT }\end{array}$ & 9 & - & - & - & - & - & - & - & $1(10)$ & $1(1.49)$ \\
\hline $\begin{array}{l}\text { C, CIP, SXT, GN, } \\
\text { F, TC, AMP, CEP, } \\
\text { CFR }\end{array}$ & 9 & - & - & - & - & - & $1(12.5)$ & - & - & $1(1.49)$ \\
\hline $\begin{array}{l}\text { C, SXT, GN, F, } \\
\text { TC, AMP, CEP, } \\
\text { CFR, CFT }\end{array}$ & 9 & $1(10)$ & - & - & - & $1(6.7)$ & - & - & $1(10)$ & $1(1.49)$ \\
\hline $\begin{array}{l}\text { CIP, SXT, GN, F, } \\
\text { TC, AMP, CEP, } \\
\text { CFR, CFT }\end{array}$ & 9 & - & - & - & - & $1(6.7)$ & - & - & - & $1(1.49)$ \\
\hline $\begin{array}{l}\text { CIP, SXT, GN, } \\
\text { TC, AMP, CEP, } \\
\text { CFR, CFT, AMI }\end{array}$ & 9 & - & - & - & - & $1(6.7)$ & - & - & - & $1(1.49)$ \\
\hline $\begin{array}{l}\mathrm{C}, \mathrm{GN}, \mathrm{F}, \mathrm{TC}, \\
\text { AMP, CEP, CFR, } \\
\text { CFT }\end{array}$ & 8 & - & - & - & - & $1(6.7)$ & - & - & - & $1(1.49)$ \\
\hline $\begin{array}{l}\text { C, SXT, F, TC, } \\
\text { AMP, CEP, CFR, } \\
\text { CFT }\end{array}$ & 8 & - & $2(25)$ & - & - & - & - & - & - & $2(2.99)$ \\
\hline $\begin{array}{l}\text { C, F, TC, AMP, } \\
\text { CEP, CFR, CFT, }\end{array}$ & 7 & - & $1(12.5)$ & - & - & - & - & - & - & $1(1.49)$ \\
\hline $\begin{array}{l}\text { C, GN, F, TC, } \\
\text { AMP, CEP, CFR }\end{array}$ & 7 & - & - & - & - & - & - & $1(16.7)$ & - & $1(1.49)$ \\
\hline $\begin{array}{l}\text { C, SXT, GN, } \\
\text { AMP, CEP, CFR, } \\
\text { CFT }\end{array}$ & 7 & - & - & - & - & - & - & - & $1(10)$ & $1(1.49)$ \\
\hline $\begin{array}{l}\text { CIP, SXT, F, } \\
\text { AMP, CEP, CFR, } \\
\text { CFT }\end{array}$ & 7 & - & $1(12.5)$ & - & - & - & - & - & - & $1(1.49)$ \\
\hline $\begin{array}{l}\text { CIP, SXT, TC, } \\
\text { AMP, CEP, CFR, } \\
\text { CFT }\end{array}$ & 7 & - & - & - & - & $1(6.7)$ & - & - & - & $1(1.49)$ \\
\hline $\begin{array}{l}\text { F, TC, TC, AMP, } \\
\text { CEP, CFR, CFT }\end{array}$ & 7 & - & - & - & - & - & - & - & $1(10)$ & $1(1.49)$ \\
\hline $\begin{array}{l}\text { GN, F, TC, AMP, } \\
\text { CEP, CFR, CFT }\end{array}$ & 7 & - & $1(12.5)$ & - & - & $1(6.7)$ & - & - & - & $2(2.99)$ \\
\hline $\begin{array}{l}\text { SXT, F, TC, AMP, } \\
\text { CEP, CFR, CFT }\end{array}$ & 7 & - & $1(12.5)$ & $1(50)$ & - & - & - & - & - & $2(2.99)$ \\
\hline $\begin{array}{l}\text { SXT, GN, F, } \\
\text { AMP, CEP, CFR, } \\
\text { CFT }\end{array}$ & 7 & $1(10)$ & - & - & - & $1(6.7)$ & - & - & - & $1(1.49)$ \\
\hline $\begin{array}{l}\text { C, F, AMP, CEP, } \\
\text { CFR, CFT }\end{array}$ & 6 & - & - & - & - & - & - & $1(16.7)$ & - & $1(1.49)$ \\
\hline
\end{tabular}


TABLE 4: Continued.

\begin{tabular}{|c|c|c|c|c|c|c|c|c|c|c|}
\hline $\begin{array}{l}\text { Resistant } \\
\text { patterns of } \\
\text { antibacterial } \\
\text { agents tested }\end{array}$ & $\begin{array}{l}\text { No. of } \\
\text { agents }\end{array}$ & $\begin{array}{c}\text { Klebsiella } \\
\text { spp., } N \\
(\%)\end{array}$ & $\begin{array}{c}\text { Pseudomonas } \\
\text { spp., } N(\%)\end{array}$ & $\begin{array}{l}\text { Acinetobacter } \\
\text { spp., } N(\%)\end{array}$ & $\begin{array}{c}\text { Enterobacter } \\
\text { spp., } N(\%)\end{array}$ & $\begin{array}{l}\text { Citrobacter } \\
\text { spp., } N(\%)\end{array}$ & $\begin{array}{c}\text { Proteus } \\
\text { spp., } N \\
(\%)\end{array}$ & $\begin{array}{l}\text { Salmonella } \\
\text { spp., } N(\%)\end{array}$ & $\begin{array}{l}\text { Escherichia } \\
\text { coli, } N(\%)\end{array}$ & $\begin{array}{l}\text { Total } \\
\text { no. }(\%)\end{array}$ \\
\hline $\begin{array}{l}\text { C, SXT, F, TC, } \\
\text { AMP, CFR }\end{array}$ & 6 & - & - & - & - & - & - & $1(16.7)$ & - & $1(1.49)$ \\
\hline $\begin{array}{l}\text { C, SXT, GN, } \\
\text { AMP, CEP, CFR }\end{array}$ & 6 & - & - & - & $1(20)$ & - & - & $1(16.7)$ & - & $2(2.99)$ \\
\hline $\begin{array}{l}\text { F, TC, AMP, CEP, } \\
\text { CFT, CFT }\end{array}$ & 6 & - & $1(12.5)$ & - & - & - & - & - & - & $1(1.49)$ \\
\hline $\begin{array}{l}\text { CIP, SXT, TC, } \\
\text { AMP, CFR }\end{array}$ & 5 & - & - & - & - & - & - & - & $2(20)$ & $1(1.49)$ \\
\hline $\begin{array}{l}\text { F, AMP, CEP, } \\
\text { CFR, CFT }\end{array}$ & 5 & $1(10)$ & - & - & - & - & - & - & - & $1(1.49)$ \\
\hline $\begin{array}{l}\text { F, TC, AMP, CEP, } \\
\text { CFR }\end{array}$ & 5 & - & - & - & - & - & - & - & - & $1(1.49)$ \\
\hline $\begin{array}{l}\text { F, TC, AMP, CFT, } \\
\text { AMI }\end{array}$ & 5 & - & - & - & - & $1(6.7)$ & - & - & - & $1(1.49)$ \\
\hline $\begin{array}{l}\text { GN, TC, AMP, } \\
\text { CFR, AMI } \\
\end{array}$ & 5 & $1(10)$ & - & - & - & - & - & - & - & $1(1.49)$ \\
\hline $\mathrm{C}, \mathrm{F}, \mathrm{T}, \mathrm{AMP}$ & 4 & - & - & - & - & - & $1(12.5)$ & - & - & $1(1.49)$ \\
\hline $\begin{array}{l}\text { F, AMP, CEP, } \\
\text { CFR }\end{array}$ & 4 & $1(10)$ & - & - & $2(40)$ & $2(13.3)$ & - & - & - & $4(5.97)$ \\
\hline $\begin{array}{l}\text { F, AMP, CFR, } \\
\text { AMI }\end{array}$ & 4 & - & - & - & $1(20)$ & - & - & - & - & $1(1.49)$ \\
\hline $\begin{array}{l}\text { F, AMP, CFR, } \\
\text { CFT }\end{array}$ & 4 & $1(10)$ & - & - & - & - & - & - & - & $1(1.49)$ \\
\hline F, T, AMP, CEP & 4 & - & - & - & - & - & - & - & - & $1(1.49)$ \\
\hline CEP, AMP, CFR & 3 & - & - & - & - & - & $1(12.5)$ & - & - & $1(1.49)$ \\
\hline F, AMP, CEP & 3 & $1(10)$ & - & - & - & $1(6.7)$ & - & - & - & $1(1.49)$ \\
\hline F, CEP, CFR & 3 & - & - & - & - & - & $1(12.5)$ & $2(33.3)$ & - & $2(2.99)$ \\
\hline F, TC, AMP & 3 & - & - & - & - & - & $1(12.5)$ & - & - & $1(1.49)$ \\
\hline SXT, F, AMP, & 3 & $1(10)$ & - & - & $1(20)$ & - & - & - & - & $2(2.99)$ \\
\hline SXT, TC, AMP & 3 & - & - & - & - & - & - & - & - & $1(1.49)$ \\
\hline $\mathrm{F}, \mathrm{TC}$ & 2 & - & - & - & - & - & $2(25)$ & - & - & $2(2.99)$ \\
\hline Total & & 10 & 8 & 2 & 5 & 15 & 8 & 6 & 10 & 64 \\
\hline
\end{tabular}

C: chloramphenicol $(30 \mu \mathrm{g})$; CIP: ciprofloxacin $(5 \mu \mathrm{g})$; SXT: trimethoprim-sulfamethoxazole; GN: gentamicin $(30 \mu \mathrm{g})$; F: nitrofurantoin; TC: tetracycline; Amp: ampicillin; Cep: cefalexin; CFR: ceftazidime; CFT: ceftriaxone; Ami: amikacin. All the tests were conducted on Mueller-Hinton agar as specified by the Clinical and Laboratory Standards Institute guidelines.

prophylaxis (ceftriaxone and ampicillin are used extensively in this setting). This issue has been identified as a major concern in the region $[4,24,25]$. Indeed, it is our opinion that the widespread use of these medications in this setting should be re-evaluated.

In the subsequent analysis of multidrug resistance by GNBs, our investigation indicates that nearly all the isolates were resistant to $>3$ classes of antibiotics. Resistance or intermediate resistance (IR) to all the antibiotics tested in this study was observed in E coli, Pseudomonas aeruginosa, Klebsiella pneumoniae, and Citrobacter spp. isolates (Table 4). Frequently, ESBL-encoding plasmids contain linked resistance determinants (class A and AmpC $\beta$-lactamases (class $\mathrm{C}$ ), among others) for the third-generation cephalosporins, SMX, tetracyclines, and aminoglycosides [10]. Resistance to this group of agents was noted in multiple isolates. In this regard, the result dovetails well with multiple reports which have noted the presence of extended and MDR isolates [23, 24]. The fact that resistance to the third-generation cephalosporins can be regarded as surrogate markers for MDR must also be noted [22].

Clearly, infection with MDR represents a formidable burden for both patients and healthcare systems. Importantly, the MDR strains and their intrinsic resistance to less costly antibiotics that are widely used in resource-limited settings like Eritrea confer limited treatment options for affected patients. Attention should also be directed at the high frequency of MDR Pseudomonas aeruginosa isolates. Many potential reservoirs of Pseudomonas aeruginosa have been identified in the hospital environment. These include cleaning solutions/disinfectants, sinks, respiratory equipment, drapes, endoscopes, physiotherapy pools, and vegetables among others. This catalogue of potential risk factors is often overlooked in infection control guidelines in SSA.

As previously described, analysis of the resistant profiles of the Staphylococcus aureus isolates was performed. Currently, pathogenic strains of Staphylococcus aureus are one of 
TABLE 5: Drug resistance patterns of Gram-positive isolates among patients in surgical wards at the Halibet and the Orotta National Referral Hospital in Asmara, Eritrea, 2017.

\begin{tabular}{lcc}
\hline Combination of drugs (\%) & No. of agents & Total no. (\%) \\
\hline C, F, CLI, ERT, P, Van, RIF & 7 & $1(5)$ \\
CIP, GN, F, ERT, Oxa, P, Van & 7 & $1(5)$ \\
C, SXT, F, Oxa, P, Van & 6 & $1(5)$ \\
C, GN, ERT, Oxa, P & 5 & $1(5)$ \\
C, TC, ERT, Oxa, P & 5 & $1(5)$ \\
CIP, GN, F, TC, Oxa & 5 & $1(5)$ \\
ERT, Oxa, P, Van, RIF & 5 & $1(5)$ \\
F, TC, Oxa, P, Van & 5 & $1(5)$ \\
F, Oxa, P, Van & 4 & $1(5)$ \\
F, P, Van, RIF & 4 & $1(5)$ \\
F, TC, P, RIF & 4 & $1(5)$ \\
Oxa, P, Van, RIF & 4 & $1(5)$ \\
CIP, Oxa, P & 3 & $1(5)$ \\
Oxa, P, Van & 3 & $2(10)$ \\
Oxa, P, Van & 2 & $1(5)$ \\
P, RIF & 2 & $1(5.3)$ \\
P, Van & 2 & $1(5.3)$ \\
P & 1 & $2(10)$ \\
Total & & $\mathbf{1 0 0}$ \\
\hline
\end{tabular}

CONS: coagulase-negative staphylococci; P: penicillin; C: chloramphenicol $(30 \mu \mathrm{g})$; CIP: ciprofloxacin; SXT: trimethoprim-sulfamethoxazole; GN: gentamicin; F: nitrofurantoin; T: tetracycline; Cli: clindamycin; ERT: erythromycin; Oxa: oxacillin; Van: vancomycin; RIF: rifampicin. All the tests were conducted on Mueller-Hinton Agar as specified by the Clinical and Laboratory Standards Institute guidelines.

the most common pathogens in hospitals worldwide. The rapidly evolving pathogenicity of Staphylococcus aureus is associated with the capacity of this pathogen to produce several virulence factors including enterotoxin serotypes A through Q (SEA-SEQ), cytolytic toxins ( $\alpha$ - and $\beta$-hemolysin), exfoliative toxins, toxic shock syndrome toxin-1 (TSST1), Panton-Valentine leukocidin (PVL), protein A, and several enzymes [19]. Apart from ceftobiprole, the presence of modified penicillin-binding protein (PBP2' or $\mathrm{PBP} 2 \mathrm{a}$ ) encoded by $\mathrm{MecA}$ confers resistance to all $\beta$ lactam antibiotics [19]. Remarkably, all the Staphylococcus aureus isolates in this study were resistant to penicillin. This phenotype has been described by several investigators in the region [13, 27-31]. The magnitude of HA-MRSA in our study was also high (70\%). High prevalence of HA-MRSA has also been reported in the region [12-14, 31, 32]. Crucially, this finding is consistent with the findings of a recent study in the same institution (MRSA prevalence $>72 \%$ ) [14]. Therefore, the high frequency of MRSA reported in these studies appears to suggest that the crisis of MDR in Eritrea is a rapidly developing problem requiring urgent attention and resolution. A quandary in need of urgent determination is whether antimicrobial susceptibility patterns observed in this location are epidemiologically related to cases in other geographic locations in the country or region? To resolve this problem, molecular characterization of isolates from specific epidemiological clusters within the country should be a principle focus.

Finally, we have to reiterate the fact that although the level of resistance to clindamycin and erythromycin was low, a concerning level of resistance was observed. This concern is particularly reinforced by the consensus that inducible resistance to clindamycin exhibited by some strains of Staphylococcus aureus warrants cautious use of this agent in the treatment of erythromycin-resistant strains. Equally concerning is the high level of resistance to vancomycin (drug of choice for HA-MRSA). The high levels of vancomycin resistance reported in this study should be treated with some caution. A foremost concern is the fact that the procedures commonly used to detect vancomycin-intermediate $S$. aureus (VISA) strains or VRSA in the regions have some limitations. More importantly, detection of these isolates should be confirmed by a reference method-a process that was not performed in this study. Regardless, we have to highlight the fact that the finding corroborates previous reports [17] and aligns with several studies in the region [12-14]. In this regard, it reinforces our previous argument that VRSA is more prevalent in parts of East Africa (Ethiopia, Eritrea, Sudan) compared with southern parts of Africa [14, 33]. More importantly, these preliminary results highlight the need for the adoption of better testing procedures for VISA or VRSA in the country.

4.1. Strength and Limitations. To our knowledge, this is the first study, performed in an internationally accredited laboratory (with requisite internal quality and external quality assurance measures) to report on the phenotypic antibiotic resistance patterns of Gram-positive and Gram-negative isolates from SSIs in two of the largest publicly funded referral hospitals in Eritrea. The data may be important in designing interventions (surveillance programs included) for prevention of SSIs in healthcare facilities and for drawing up effective therapeutic guidelines. However, the study had several limitations including failure to perform anaerobic culture and molecular characterization of the isolates. Another notably finding which should be treated with a lot of caution is the high resistance to vancomycin. Regarding this concern, we have to emphasize the fact that the disk diffusion procedure used in this study or in most studies from the region has limitations in detecting resistance and that detection of these isolates should be confirmed by a reference method. In addition, sensitivity to specific drugs such as linezolid, piperacillin/tazobactam, and carbapenems (e.g., imipenem), among others, was not evaluated.

\section{Conclusion}

In this study, Citrobacter spp., Klebsiella spp., Escherichia coli, Proteus spp., Pseudomonas aeruginosa, Salmonella spp., Enterobacter spp., and Acinetobacter spp. were the most common isolates. In contrast, the predominant Grampositive isolates included Staphylococcus aureus, CONS, and Streptococcus viridians. Furthermore, it is clear that the significant exposure of patients to MDR including HAMRSA and VRSA represents a significant problem for countries like Eritrea. Much work is needed in Eritrea to formulate stricter policies and guidelines on infection control practices (decontamination of surfaces, 
antimicrobial surveillance, antibiotic stewardship, cohorting and decolonization of patients and healthcare workers, etc.). Concomitant improvement and expansion/decentralization of diagnostic services and establishment of a central organizing body to routinely collate, analyze, and report relevant data should also be considered.

\section{Abbreviations}

AST: Antimicrobial susceptibility testing

CLSI: Clinical and Laboratory Standards Institute

ESBL: $\quad$ Extended spectrum beta lactamases

GNB: Gram negative bacteria

G3CREC: Third-generation cephalosporin-resistant Escherichia coli

GNB: Gram-negative bacteria

MDR: Multidrug resistance

HA- Hospital-acquired methicillin-resistant

MRSA: Staphylococcus aureus

SSI: $\quad$ Surgical site infection

VRSA: Vancomycin-resistant Staphylococcus aureus

TSST-1: Toxic shock syndrome toxin-1

PVL: $\quad$ Panton-Valentine.

\section{Data Availability}

All data sets used for this study are available from the corresponding author on reasonable request.

\section{Conflicts of Interest}

The authors declare that they have no conflicts of interest.

\section{Acknowledgments}

The authors thank the Eritrean Ministry of Health and all the patients who participated in this study. The authors thank the staff of Eritrean National Health Laboratory (NHL) and health workers in the surgical department for their excellent technical assistance.

\section{References}

[1] J. P. Burke, "Infection control-a problem for patients safety," New England Journal of Medicine, vol. 348, p. 7, 2003.

[2] B. Mawalla, S. E. Mshana, P. L. Chalya, C. Imirzalioglu, and W. Mahalu, "Predictors of surgical site infections among patients undergoing major surgery at Bugando Medical Centre in Northwestern Tanzania," BMC Surgery, vol. 11, p. 21, 2011.

[3] W. J. Martone and R. L. Nichols, "Recognition, prevention, surveillance, and management of surgical site infections: introduction to the problem and symposium overview," Clinical Infectious Diseases, vol. 33, no. s2, pp. S67-S68, 2001.

[4] J. Manyahi, M. I. Matee, M. Majigo, S. Moyo, S. E. Mshana, and E. F. Lyamuya, "Predominance of multi-drug resistant bacterial pathogens causing surgical site infections in Muhimbili national hospital, Tanzania," BMC Research Notes, vol. 7, p. 500, 2014.

[5] F. Perez, A. M. Hujer, K. M. Hujer, B. K. Decker, P. N. Rather, and R. A. Bonomo, "Global challenge of multidrug-resistant
Acinetobacter baumannii," Antimicrobial Agents and Chemotherapy, vol. 51, no. 10, pp. 3471-3484, 2007.

[6] G. G. Zhanel, M. DeCorby, H. Adam et al., "Prevalence of antimicrobial-resistant pathogens in Canadian hospitals: results of the Canadian ward surveillance study (CANWARD 2008)," Antimicrobial Agents and Chemotherapy, vol. 54, no. 11, pp. 4684-4693, 2010.

[7] P. Agaba, J. Tumukunde, J. V. B. Tindimwebwa, and A. Kwisera, "Nosocomial bacterial infections and their antimicrobial susceptibility patterns among patients in Ugandan intensive care units: a cross sectional study," BMC Research Notes, vol. 10, p. 349, 2017.

[8] J. Seni, C. F. Najjuka, D. P. Kateete et al., "Antimicrobial resistance in hospitalized surgical patients: a silently emerging public health concern in Uganda," BMC Research Notes, vol. 6, p. 298, 2013.

[9] D. Maina, G. Omuse, G. Revathi, and R. D. Adam, "Spectrum of microbial diseases and resistance patterns at a private teaching hospital in Kenya: implications for clinical practice," PLoS One, vol. 11, no. 1, p. e0147659, 2016.

[10] M. E. Levison, "Plasmid-mediated extended-spectrum $\beta$-lactamases in organisms other than Klebsiella pneumoniae and Escherichia coli: a hidden reservoir of transferable resistance genes," Current Infectious Disease Reports, vol. 4, no. 3, p. 181, 2002.

[11] G. G. Zhanel, M. Decorby, N. Laing et al., "Antimicrobialresistant pathogens in intensive care units in Canada: results of the Canadian national intensive care unit (CAN-ICU) study, 2005-2006, 2008-2006," Antimicrobial Agents and Chemotherapy, vol. 52, no. 4, pp. 1430-1437, 2008.

[12] S. Eshetie, F. Tarekegn, F. Moges, A. Amsalu, W. Birhan, and K. Huruy, "Methicillin resistant Staphylococcus aureus in Ethiopia: a meta-analysis," BMC Infectious Diseases, vol. 16, no. 1, p. 689, 2016.

[13] G. Godebo, G. Kibru, and H. Tassew, "Multidrug-resistant bacterial isolates in infected wounds at jimma university specialized hospital, Ethiopia," Annals of Clinical Microbiology and Antimicrobials, vol. 12, no. 1, p. 17, 2013.

[14] E. Y. Garoy, Y. B. Gebreab, O. O. Achila et al., "Methicillinresistant Staphylococcus aureus (MRSA): prevalence and antimicrobial sensitivity pattern among patients-A multicenter study in Asmara, Eritrea," Canadian Journal of Infectious Diseases and Medical Microbiology, vol. 2019, Article ID 8321834, 9 pages, 2019.

[15] N. S. Levine, R. B. Lindberg, A. D. Mason Jr, and B. A. Pruitt Jr, "The quantitative swab culture and smear," The Journal of Trauma: Injury, Infection, and Critical Care, vol. 16, no. 2, pp. 89-94, 1976.

[16] Clinical and Laboratory Standards Institute [CLSI], Performance Standards for Antimicrobial Susceptibility Testing; Supplement M100, Clinical and Laboratory Standards Institute, Wayne, PA, USA, 27th Edn. edition, 2017.

[17] A.-P. Magiorakos, A. Srinivasan, R. B. Carey et al., "Multidrugresistant, extensively drug-resistant and pandrug-resistant bacteria: an international expert proposal for interim standard definitions for acquired resistance," Clinical Microbiology and Infection, vol. 18, no. 3, pp. 268-281, 2012.

[18] M. E. Falagas, J. Karageorgopoulos, Leptidis, and I. P. Korbila, "MRSA in Africa: filling the global map of Antimicrobial resistance," Plos One, vol. 8, no. 7, p. e68024, 2013.

[19] S. Monecke, G. Coombs, A. C. Shore et al., "A field guide to pandemic, epidemic and sporadic clones of methicillin-resistant Staphylococcus aureus," PLoS One, vol. 6, no. 4, p. e17936, 2011. 
[20] N. Moremi, M. F. Mushi, M. Fidelis, P. Chalya, M. Mirambo, and S. E. Mshana, "Predominance of multi-resistant gramnegative bacteria colonizing chronic lower limb ulcers (CLLUs) at Bugando Medical Center," BMC Research Notes, vol. 7, no. 1, p. 211, 2014

[21] W. Mulu, G. Kibru, and M. Damtie, "Postoperative nosocomial infections and antimicrobial resistance pattern of bacteria isolates among patients admitted at Felege Hiwot referral hospital, Bahirdar, Ethiopia," Ethiopian Jounal of Health Sciences, vol. 22, no. 10, pp. 7-18, 2012.

[22] M. E. A. de Kraker, P. G. Davey, and H. Grundmann, "Mortality and hospital stay associated with resistant Staphylococcus aureus and Escherichia coli bacteremia: estimating the burden of antibiotic resistance in europe," PLoS Medicine, vol. 8, no. 10, p. e1001104, 2011.

[23] J. R. Anguzu and D. Olila, "Drug sensitivity patterns of bacterial isolates from septic post-operative wounds in a regional referral hospital in Uganda," African Health Sciences, vol. 7, no. 3, pp. 148-154, 2007.

[24] R. E. Mangesha, B. G.-S. Kasa, M. Saravan, D. F. Berhe, and A. G. Wasihun, "Aerobic bacteria in post surgical wound infections and pattern of their antimicrobial susceptibility in Ayder Teaching and Referral Hospital, Mekelle, Ethiopia," BMC Research Notes, vol. 7, p. 575, 2014.

[25] S. E. Mshana, M. Matee, and M. Rweyemamu, "Antimicrobial resistance in human and animal pathogens in Zambia, Democratic Republic of Congo, Mozambique and Tanzania: an urgent need of a sustainable surveillance system," Annals of Clinical Microbiology and Antimicrobials, vol. 12, no. 1, p. 28, 2013.

[26] C. A. Muto, J. A. Jernigan, B. E. Ostrowsky et al., "SHEA guideline for preventing nosocomial transmission of multidrug-resistant strains of Staphylococcus aureus and enterococcus," Infection Control \& Hospital Epidemiology, vol. 24, no. 5, pp. 362-386, 2003.

[27] K. Wangai, M. M. Masika, M. C. Maritim, and R. A. Seaton, "Methicillin-resistant Staphylococcus aureus (MRSA) in East Africa: red alert or red herring?," BMC Infectious Diseases, vol. 19, no. 1, p. 596, 2019.

[28] T. Kejela and K. Bacha, "Prevalence and antibiotic susceptibility pattern of methicillin-resistant Staphylococcus aureus (MRSA) among primary school children and prisoners in Jimma Town, Southwest Ethiopia," Annals of Clinical Microbiology and Antimicrobials, vol. 12, no. 1, p. 11, 2013.

[29] S. I. Kheder, N. A. Ali, and A. I. Fathelrahman, "Prevalence and antimicrobial susceptibility pattern of methicillin resistance Staphylococcus in a Sudanese surgical ward," Pharmacology \& Pharmacy, vol. 3, no. 1, pp. 103-108, 2012.

[30] H. Legese, A. G. Kahsay, A. Kahsay et al., "Nasal carriage, risk factors and antimicrobial susceptibility pattern of methicillin resistant Staphylococcus aureus among healthcare workers in Adigrat and Wukro hospitals, Tigray, Northern Ethiopia," BMC Research Notes, vol. 11, p. 250, 2018.

[31] M. A. E. Elimam, S. Rehan, M. A. Elmekki, and MM. Elhassan, "Emergence of vancomycin resistant and methcillin resistant staphylococus aureus in patients with different clinical manifestations in Khartoum state, Sudan," Journal of America Science, vol. 10, no. 6, 2014.

[32] E. K. Maina, C. Kiiyukia, C. N. Wamae, P. G. Waiyaki, and S. Kariuki, "Characterization of methicillin-resistant Staphylococcus aureus from skin and soft tissue infections in patients in Nairobi, Kenya," International Journal of Infectious Diseases, vol. 17, no. 2, pp. e115-e119, 2013.
[33] A. M. Aiken, I. M. Mutuku, A. J. Sabat et al., "Carriage of Staphylococcus aureus in thika level 5 hospital, Kenya: a cross-sectional study," Antimicrobial Resistance and Infection Control, vol. 3, p. 22, 2014. 